\title{
Influence of Compatibilizer and Processing Conditions on Morphology, Mechanical Properties, and Deformation Mechanism of PP/Clay Nanocomposite
}

\author{
B. Akbari ${ }^{1,2}$ and R. Bagheri ${ }^{1}$ \\ ${ }^{1}$ Polymeric Materials Research Group, Department of Materials Science and Engineering, Sharif University of Technology, \\ P.O. Box 11155-9466, Tehran 11365, Iran \\ ${ }^{2}$ Materials Engineering and Research, SAPCO, P.O. Box 13885-315, Tehran, Iran
}

Correspondence should be addressed to R. Bagheri, rezabagh@sharif.edu

Received 15 December 2011; Revised 31 January 2012; Accepted 14 February 2012

Academic Editor: Luiz Antonio Ferreira Coelho

Copyright ( 2012 B. Akbari and R. Bagheri. This is an open access article distributed under the Creative Commons Attribution License, which permits unrestricted use, distribution, and reproduction in any medium, provided the original work is properly cited.

\begin{abstract}
Polypropylene/montmorillonite nanocomposite was prepared by melt intercalation method using a twin-screw extruder with starve feeding system in this paper. The effects of compatibilizer, extruder rotor speed and feeding rate on properties of nanocomposite were investigated. Structure, tensile, and impact properties and deformation mechanism of the compounds were studied. For investigation of structure and deformation mechanisms, X-ray diffraction (XRD) and transmission optical microscopy (TOM) techniques were utilized, respectively. The results illustrate that introduction of the compatibilizer and also variation of the processing conditions affect structure and mechanical properties of nanocomposite.
\end{abstract}

\section{Introduction}

Inorganic fillers are extensively used in plastics industry in order to develop some properties such as stiffness, hardness, heat deflection temperature, and shrinkage along with cost decrease. The effects of filler particles on composite properties mainly depend on their shape, size, surface characterization, and extent of dispersion. In general, mechanical properties of polymers which are reinforced with microparticles are lower than those of nanocomposites [1]. In addition, obtaining desired physical properties such as surface roughness and permeability is difficult when microparticles are incorporated [1]. This is why many researches have focused on developing nanocomposites in recent years [2-4]. Among different nanofillers used, layered silicates such as montmorillonite have been considered significantly in recent years [5-12]. Polymers reinforced with montmorillonite reveal improvement of both mechanical and physical properties including elastic modulus, strength, heat distortion temperature, and permeability [5].

Use of layered silicates in polymeric matrices was started early in 90s by introduction of PA/nanoclay [7]. Since then, many polymers have been examined in such composites. Among them, polypropylene has been considered significantly [12]. This is due to the high growth rate of this polymer, especially in autoindustry [12]. It is well known that in such a nonpolar resin, use of a kind of compatibilizer along with high shear stress is required to obtain a reasonable dispersion of clay platelets [13]. Please note that the nonpolar nature of polypropylene may even suppress intercalation of clay in the absence of compatibilizer [14]. In this case, nanocomposite will not form, and improved properties are not obtained. Many efforts have been conducted to improve dispersion of clay in polypropylene using functional oligomers as compatibilizer [14-22]. For this purpose, it is necessary to graft a polar compound such as maleic anhydride to the main chain of the nonpolar polymer. This creates a functional polymer which is miscible in the matrix, while it is compatible with clay as well. The effectiveness of $\mathrm{PP}-\mathrm{g}-\mathrm{MA}$ as compatibilizer in $\mathrm{PP} /$ clay system depends on its molecular weight and MA content which determines its functionality [20]. The effect of MA content on dispersion of clay in the matrix has been investigated notably, but the 
results are to some extent contradictory [14-16, 18, 20-22]. The high amount of MA usually enhances the diffusion of polypropylene chains between the silicate layers, although it may lead to immiscibility of compatibilizer in the matrix, and thus, resulting in reduction of mechanical properties of the composite [14]. Reichert et al. [18], however, found that at a given clay loading, high MA content, that is, $4.2 \mathrm{wt} . \%$, enhanced mechanical strength further than that of the low MA content, that is, $2.9 \mathrm{wt} \%$. Nevertheless, the influence of molecular weight of the compatibilizer is less obvious [20]. Most researchers have used low molecular weight PP oligomers for grafting to MA to improve the diffusion of chains into clay galleries $[14-16,18]$. On the other hand, Wang et al. [21] illustrated that although low molecular weight $\left(M_{w}=9100 \mathrm{~g} / \mathrm{mol}\right)$ oligomer resulted in better dispersion, high molecular weight $\left(M_{w}=330,000 \mathrm{~g} / \mathrm{mol}\right)$ compatibilizer gave rise to better enhancement in mechanical properties. Kim et al. [22] incorporated four PP compatibilizers with different degrees of MA grafting and three PP matrices with low, medium, and high viscosity. They found that the system containing the low viscosity PP and the least MA content compatibilizer resulted in the greatest enhancement of dynamic storage modulus [22]. Therefore, it is claimed that without mentioning the molecular weight of compatibilizer, it is hard to judge the importance of the viscosity of individual components [22].

The role of processing conditions on properties of PP based nanocomposites is the subject of very few researches [20, 23-26]. Up to now, the literature contains no summary conclusions about the optimum processing conditions for obtaining desired properties of polymeric nanocomposites. Some works on the influence of processing conditions on the properties of the nanocomposites have been executed [2326]. Dennis et al. [23] studied polyamide nanocomposite and showed that the surface chemistry of the clay platelets and the extent of shear stress determine the structure and, thus, the properties of the nanocomposite. Incarnato et al. [24] studied the effect of the extrusion rate on the properties of polyamide 6-based nanocomposites. Wang et al. [20] focused on the influence of the compatibilizer and melt mixing temperature on the dispersion of clay in polypropylene. They found that higher mixing temperature $\left(160^{\circ} \mathrm{C}\right.$ instead of $150^{\circ} \mathrm{C}$ ) enhances diffusion of molecular chains into galleries but reduces shear viscosity. Therefore, low viscosity does not make enough shear for breaking up the platelets and leads to poor exfoliation morphology [20]. Modesti et al. [25] analyzed the effect of temperature profile and screw speed of extruder on the structure of nanocomposites. Their results showed that the applied shear stress is much more effective on the morphology (the extent of intercalation/exfoliation) than the residence time [25]. In addition, Lertwimolnun and Vergnes [26] characterized the role of mixing temperature, rotor speed, and mixing time in both internal mixer and extruder. They stated that increasing shear stress and mixing time and decreasing compounding temperature improve the extent of exfoliation [26].

In this research, PP-g-MA has been made using the same polymer used as the matrix. Influences of such a compatibilizer and processing conditions including rotor speed and feeder rate on morphology, mechanical properties, and deformation mechanism of polypropylene/clay nanocomposite have been investigated.

\section{Experimental Method}

2.1. Materials. A kind of isotactic polypropylene with the MFI (at $230^{\circ} \mathrm{C}, 2.16 \mathrm{~kg}$ ) of $8 \mathrm{gr} / 10 \mathrm{~min}$ by the trade name of PI080 from Imam Khomeini Petrochemical Complex and an organophilic montmorillonite with the CEC of 110$120 \mathrm{meq} / 100 \mathrm{~g}$ by the trade name of NANOLIN DK4 from FCC were used. In order to synthesize compatibilizer, maleic anhydride (MA), and dicumyl peroxide (DCP), as initiator, both from Merck were incorporated.

2.2. Preparation of Nanocomposites. The processing conditions for preparing different nanocomposites made are summarized in Table 1. In order to make nanocomposite without compatibilizer, mixtures of $1.5,3$, and $5 \mathrm{wt} . \%$ nanoclay with polypropylene were blended in a corotating twin-screw extruder $(\mathrm{L} / \mathrm{D}=20)$ at a rotor speed of 50 and $100 \mathrm{rpm}$, while the temperatures in the successive zones were set at $140,170,180$, and $190^{\circ} \mathrm{C}$.

For preparing the compatibilizer, MA (2 phr) and DCP (0.1 phr) were dissolved in acetone and physically mixed with PP pellets at room temperature. After evaporation of acetone in air, MA and DCP precipitate on the pellets' surface homogeneously. As mentioned earlier, polypropylene used for making compatibilizer was the same polymer used as the matrix [23].

In the case of compatibilized nanocomposites, again, 1.5, 3 , and 5 wt. $\%$ clay samples were made. The magnitude of compatibilizer in each case was equal to the clay loading. Temperature regimes in extrusion of compatibilized systems were similar to those of the noncompatibilized ones. Injection molding was utilized to make samples of the formulations made.

2.3. Characterization Techniques. Wide-angle X-ray diffraction (XRD) analyses were conducted using a Bruker Axs generator. An acceleration voltage of $40 \mathrm{kV}$ and a current of $30 \mathrm{~mA}$ were applied using $\mathrm{Cu}-\mathrm{K} \alpha$ radiation. Tensile tests were performed using a universal testing frame (Hounsfield H10KS) equipped with an extensometer, according to ASTM D638. Notched impact strengths were measured according to ASTM D256 using a $5 \mathrm{~J}$ impact hammer from Santam. The dimensions of Charpy samples were $25 \times 12.5 \times$ $3.5 \mathrm{~mm}^{3}$. All mechanical tests were performed on average number of 5 specimens. Study of deformation behavior at the notch tip was done on thin samples cut from threepoint-bending (3PB) specimens using a transmitted light optical microscope (OLYMPUS DL12). In order to do this experiment, notched Charpy samples were subjected to a certain amount of loading $(215 \mathrm{~N})$ in $3 \mathrm{~PB}$ mode, in which a well-developed damage zone forms at the notch tip of samples. For observation of the deformation mechanism, the samples were cut and polished from both sides to reach the thickness of approximately less than 100 micrometers in the 
TABLE 1: The compounds prepared in this study.

\begin{tabular}{|c|c|c|}
\hline \multirow{2}{*}{ Compound } & \multicolumn{2}{|c|}{ Processing conditions } \\
\hline & $\begin{array}{l}\text { Rotor speed } \\
\quad(\mathrm{rpm})\end{array}$ & $\begin{array}{c}\text { Feeder speed } \\
\quad(\mathrm{rpm})\end{array}$ \\
\hline (1) PP + Clay & 50 & 10 \\
\hline (2) PP + Clay & 100 & 10 \\
\hline (3) $\mathrm{PP}+\mathrm{PP}-\mathrm{g}-\mathrm{MA}+$ Clay & 50 & 10 \\
\hline (4) $\mathrm{PP}+\mathrm{PP}-\mathrm{g}-\mathrm{MA}+$ Clay & 100 & 10 \\
\hline (5) $\mathrm{PP}+\mathrm{PP}-\mathrm{g}-\mathrm{MA}+$ Clay & 100 & 15 \\
\hline (6) $\mathrm{PP}+\mathrm{PP}-\mathrm{g}-\mathrm{MA}+$ Clay & 150 & 15 \\
\hline
\end{tabular}

TABLE 2: XRD results of organoclay and samples made (without compatibilizer).

\begin{tabular}{lcc}
\hline Sample* & $2 \theta$ & $d$ Spacing $(\AA)$ \\
& $(001)$ & $(001)$ \\
\hline Organoclay & 2.58 & 34.23 \\
$(1)$ & 2.55 & 34.61 \\
$(2)$ & 2.63 & 33.56 \\
\hline
\end{tabular}

* The numbers in this column correspond to the compounds introduced in Table 1.

middle of the sample. This approach is further discussed in [27].

\section{Results and Discussion}

3.1. Characterization of the Structure. The results of XRD for organoclay and $\mathrm{PP} / 5$ wt.\% clay nanocomposites with regards to different rotor speeds have been shown in Figure 1 and Table 2.

As can be seen in Table 2, incorporation of organoclay into polypropylene at the rotor speed of $50 \mathrm{rpm}$, that is, Sample (1) in Table 2, results in slight increased $d$-spacing of (001) planes. Table 2 also illustrates that the increase of rotor speed to $100 \mathrm{rpm}$, that is, Sample (2), will result in reduction of the $d$-spacing of the same planes. In order to explain this observation, one should consider the fact that the nanostructure in such a composite depends on both shear stress and the diffusion rate [23]. Please note that it is necessary to apply sufficient shear stress and allocate enough time to the polymer chains in order to be diffused into the galleries. At the rotor speed of $50 \mathrm{rpm}$, that is, Sample (1) in Table 2, it seems that the time for diffusion of the polymer chains into the galleries is sufficient. Thus, an increase in $d$-spacing is observed (Table 2). However, the value of $d$ spacing in Sample (1) is not large enough that might be as a result of the lack of compatibility between the polymer and the organoclay. This subject will be further examined later in this paper.

Sample (2) which corresponds to the rotor speed of $100 \mathrm{rpm}$ illustrates reduction in $d$-spacing compared to that of Sample (1), that is, rotor speed of $50 \mathrm{rpm}$. This reduction in $d$-spacing of $\mathrm{PP} /$ clay nanocomposites during no incorporation of any compatibilizer was reported in literature before $[14,18,20,21]$. The reason for this phenomenon

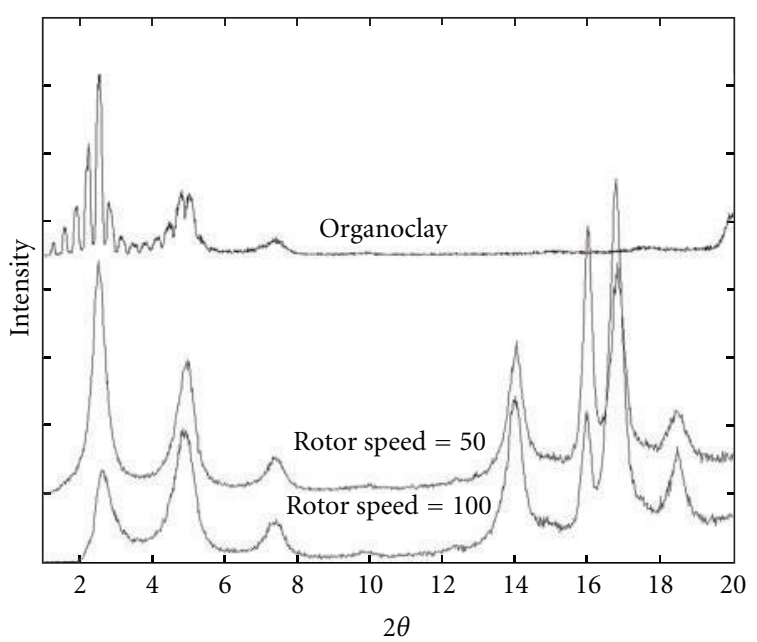

FIGURE 1: XRD graphs of organoclay and polypropylene based nanocomposites without compatibilizer compounded with Feeder Speed $=10 \mathrm{rpm}$.

has been attributed to the incompatibility of the polar hydroxyl groups on the surface of the clay platelets and the nonpolar polypropylene chains based on studies done in those researches $[14,18,20,21]$. It seems that the incompatibility of clay and PP is not the only reason for decreasing the space between the layers. The slight increase of $d$-spacing in the condition of $50 \mathrm{rpm}$ is an evidence for this claim. It is possible that the polypropylene chains stick to the surface modifier of clay (alkyl ammonium ions in this work), which is compatible with polymer during mixing. If the time for diffusing the polymer chains into layers is sufficient (Rotor Speed $=50 \mathrm{rpm}$ ), some of them may penetrate into layers (the $d$-spacing will increase). However, if there is no sufficient time for diffusing (Rotor Speed $=100 \mathrm{rpm}$ ), it seems that not only polymeric molecules are not able to penetrate into layers, but also they may pull out some surface modifier of clay during mixing (decreasing in $d$-spacing of layers will happen). Of course, it is worth mentioning that the sufficient time for diffusing the polymer chains into layers is different under conditions of with/without compatibilizer. Certainly, this argumentation is only a hypothesis. In other words, this matter is justified by several evidences, reasons, references, and so forth and it has been stated here only as a presumption.

The results of XRD relate to organoclay and $\mathrm{PP} /$ clay nanocomposites with compatibilizer in different rotor speeds, and feeder speeds have been shown in Figure 2 and Table 3.

A comparison of Tables 2 and 3 demonstrates the influence of compatibilizer on the structure of PP/clay nanocomposites, that is, increase in $d$-spacing when compatibilizer is incorporated. Figure 3 has been displayed for easier evaluating of the role of rotor speed played about the structure of compounds. As can be seen in Figure 3, the amounts of $2 \theta$ are changed to the right with the increased speed of the rotor of the extruder in the composite with compatibilizer (Sample (3), i.e., rotor speed of $50 \mathrm{rpm}$, and Sample (4), i.e., rotor 


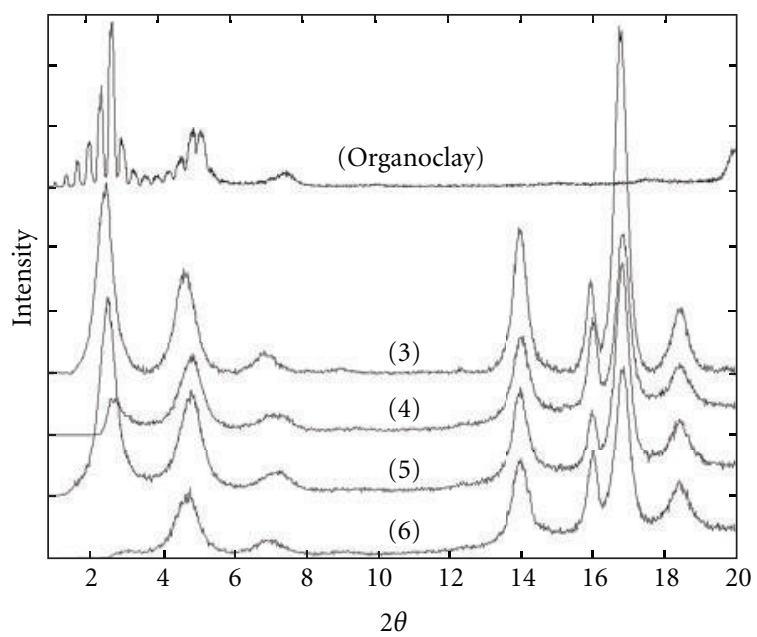

FIGURE 2: XRD graphs of organoclay and polypropylene based nanocomposites with compatibilizer. Numbers (3)-(6) correspond to the systems introduced in Table 1.

TABLE 3: XRD results of organoclay and samples made (with compatibilizer) in different processing conditions as shown in Figure 2.

\begin{tabular}{lcc}
\hline Sample & $2 \theta$ & $d$ Spacing $(\AA)$ \\
$(001)$ & $(001)$ \\
\hline Organoclay & 2.58 & 34.23 \\
$(3)$ & 2.41 & 36.62 \\
$(4)$ & 2.57 & 34.34 \\
$(5)$ & 2.43 & 36.32 \\
$(6)$ & - & - \\
\hline
\end{tabular}

speed of $100 \mathrm{rpm}$ ) like without compatibilizer (Sample (1), i.e., rotor speed of $50 \mathrm{rpm}$, and Sample (2), i.e., rotor speed of $100 \mathrm{rpm}$ ). The change from $2 \theta$ peak to the right means that the intergallery spacing of layers has been decreased as shown in Tables 2 and 3 related to Samples (1) to (4). Also, as seen in Figure 3, the peak intensity at (001) planes has notably decreased under the condition of $100 \mathrm{rpm}$ compared to $50 \mathrm{rpm}$ in both conditions of incorporating compatibilizer (Sample (3), i.e., rotor speed of $50 \mathrm{rpm}$, and Sample (4), i.e., rotor speed of $100 \mathrm{rpm}$ ) and without compatibilizer (Sample (1), i.e., rotor speed of $50 \mathrm{rpm}$, and Sample (2), i.e., rotor speed of $100 \mathrm{rpm}$ ). Because the peak intensity at (001) planes is related to the number of layers in stacks of clay, these results demonstrate that the average number of platelets in stacks has decreased by increasing the shear stress on the system.

In addition, Table 3 illustrates that the space between layers has been increased by incorporating of compatibilizer in both conditions (rotor Speed of 50, i.e., Sample (3) and rotor speed of $100 \mathrm{rpm}$, i.e., Sample (4)). Of course, because of more residence time, increasing the $d$-spacing of clay in rotor speed of $50 \mathrm{rpm}$, that is, Sample (3), is more than $100 \mathrm{rpm}$, that is, Sample (4).

The effects of rotor speed and feeder speed on the dispersion of nanoclay in a polypropylene matrix have been investigated before by using an internal mixer and an extruder,

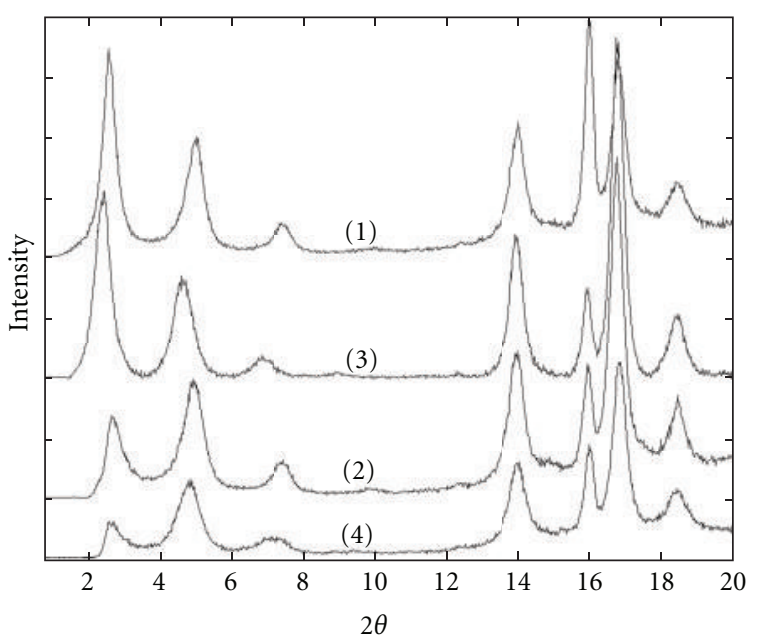

Figure 3: XRD graphs of compounds: (1)-(4).

respectively [26]. These researchers observed that the space between layers did not significantly depend on the shear rate or feeder speed [26]. On the contrary, they showed that the extent of exfoliation increased by the increase of the rotor speed of the internal mixer or with decreasing the feeder speed of the extruder [26]. The authors stated that increasing the feeder speed decreased the residence time. Therefore, it decreased the degree of exfoliation [26]. In the internal mixer, contrary to that of the extruder, the variation of rotor speed did not affect the residence time. Thus, this did not influence the $d$-spacing [26]. As can be seen in Figure 2, increasing the rotor speed in both conditions, 50 to $100 \mathrm{rpm}$, that is, Samples (3) and (4), and 100 to $150 \mathrm{rpm}$, that is, Samples (5) and (6) would result in reducing the peak intensity at (001) planes; that is, it increased the degree of exfoliation. This shows that under the conditions of this experiment, the shear rate is a more important factor regarding the degree of exfoliation than the residence time. Figure 2 and Table 3 reveal the influence of feeder rate on the structure of nanocomposite. As observed, with increasing the feeder rate from 10, that is, Sample (4) to 15, that is, Sample (5) the $2 \theta$ of the layers has decreased which is a symptom of increasing the $d$-spacing of (001) planes. At the same time, increasing the feeder rate has reduced the degree of exfoliation (has increased the peak intensity at (001) planes). Please note that with increasing the feeder rate at constant rotor speed, the intergallery spacing has been increased despite the reduction of the residence time. This means that as well as residence time, the effective $L / D$ ratio has also been affected by the feeder rate in starve feeding [28]. It seems that increasing the feeder rate of extruder will increase the effective $L / D$ ratio. Therefore, the forces, the velocity of conveyed material, and the amount of deformation will be changed [29].

On the other hand, the minimum strain $\left(\gamma_{0}\right)$, the mean total strain $(\bar{\gamma})$ and the strain distribution function $(F(\gamma))$, and the terms of applied strain to the melt of polymer are proportional to $L / H$ in mixing zone $(H$ is the distance between cylinder and screw in extruder) [30]. It means 
TABLE 4: Mechanical properties of PP/Clay samples in different conditions.

\begin{tabular}{lcccccccc}
\hline Sample & $\begin{array}{c}\text { PP } \\
(\mathrm{wt} \%)\end{array}$ & $\begin{array}{c}\text { PPgMA } \\
(\mathrm{wt} \%)\end{array}$ & $\begin{array}{c}\text { Clay } \\
(\mathrm{wt} \%)\end{array}$ & $\begin{array}{c}\text { Rotor speed } \\
(\mathrm{rpm})\end{array}$ & $\begin{array}{c}\text { Feeder speed } \\
(\mathrm{rpm})\end{array}$ & $\begin{array}{c}\text { Elastic modulus } \\
(\mathrm{MPa})\end{array}$ & $\begin{array}{c}\text { Yield stress } \\
(\mathrm{MPa})\end{array}$ & $\begin{array}{c}\text { Impact strength } \\
\left(\mathrm{KJ} / \mathrm{m}^{2}\right)\end{array}$ \\
\hline & 100 & - & - & - & - & 1588 & 28.7 & 2.2 \\
$(1)$ & 98.5 & - & 1.5 & 50 & 10 & 1792 & 30.1 & 3.1 \\
$(1)$ & 97 & - & 3 & 50 & 10 & 1891 & 30.0 & 3.6 \\
$(1)$ & 95 & - & 5 & 50 & 10 & 1952 & 29.4 & 3.6 \\
$(2)$ & 98.5 & - & 1.5 & 100 & 10 & 1711 & 29.3 & 2.9 \\
$(2)$ & 97 & - & 3 & 100 & 10 & 1842 & 29.9 & 2.9 \\
$(2)$ & 95 & - & 5 & 100 & 10 & 1871 & 29.2 & 3.5 \\
$(3)$ & 97 & 1.5 & 1.5 & 50 & 10 & 1804 & 30.1 & 2.5 \\
$(3)$ & 94 & 3 & 3 & 50 & 10 & 1929 & 30.2 & 3.3 \\
$(3)$ & 90 & 5 & 5 & 50 & 10 & 2032 & 30.0 & 3.4 \\
$(4)$ & 97 & 1.5 & 1.5 & 100 & 10 & 1787 & 30.2 & 2.8 \\
$(4)$ & 94 & 3 & 3 & 100 & 10 & 1822 & 29.5 & 3.3 \\
$(4)$ & 90 & 5 & 5 & 100 & 10 & 1918 & 28.9 & 3.3 \\
$(5)$ & 90 & 5 & 5 & 100 & 15 & 1931 & 29.8 & 3.3 \\
$(6)$ & 90 & 5 & 5 & 150 & 15 & 1961 & 30.1 & 3.0 \\
\hline
\end{tabular}

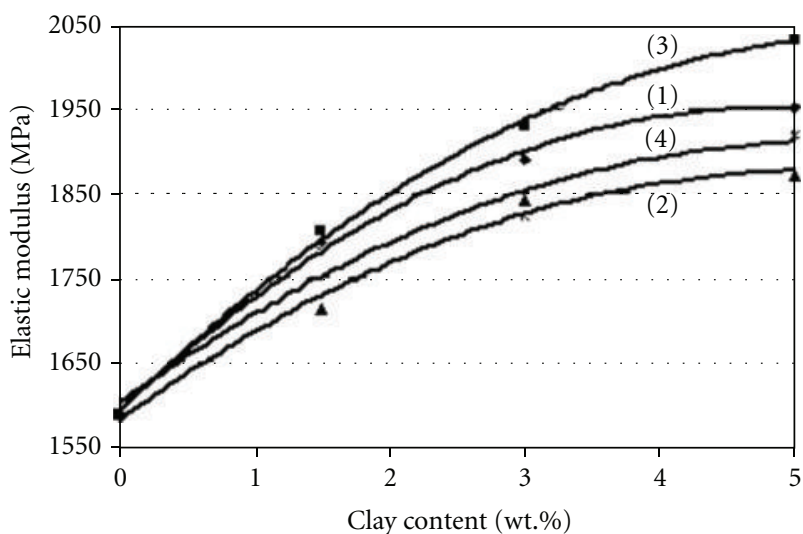

FIGURE 4: Variation of elastic modulus versus weight percentage of clay in the samples (1)-(4).

that with increasing $L / H$, the minimum and the mean total strains applied to the melt in the mixing zone increase. According to these explanations, the formation of the structure of nanocomposites in different feeder rates can be clarified (samples (4) and (5) in Figure 2 and Table 3). One may conclude that due to shorter mean residence time of material in the extruder, the peak intensity at (001) planes is enhanced, and due to higher minimum and mean total strains applied to the mixture, the $d$-spacing of the layers is increased, during the time of application of higher feeder rate, that is, increasing from 10 to $15 \mathrm{rpm}$.

3.2. Mechanical Properties. The results of the measurements of mechanical properties are illustrated in Table 4.

The effect of the compatibilizer and the rotor speed on the elastic modulus of PP/clay nanocomposites are illustrated in Figure 4.
As can be seen in Figure 4, the elastic modulus increases with decreasing the rotor speed, that is, Sample (1) compared with Sample (2), and the existence of compatibilizer, that is, Sample (4) compared with Sample (2) and Sample (3) compared with Sample (1). These observations can be explained by considering the structure of the samples $(d-$ spacing of (001) layers). In Sample 2 (Figure 4 and Table 2) the amount of $d$-spacing reveals that molecular chains of PP have not been diffused into the clay galleries. Therefore, the system acts like conventional composites. In the case of using compatibilizer, that is, Sample (4), the $d$-spacing of layers have slightly increased in comparison with organoclay, and proportionally, the aspect ratio of the reinforcement has been increased. Therefore, it is probable that elastic modulus slightly increases under such a condition. This, together with possible stronger adhesion between clay and polymer due to incorporating compatibilizer can explain the higher modulus of Samples (1) and (3).

Based on these results, one may claim that there are two structural parameters which affect elastic modulus of nanocomposites: the $d$-spacing of layers (the aspect ratio of layers) and the number of layers in each stack (the aspect ratio of the stacks). Comparing the results of Samples (3) and (4) (Figures 3 and 4 and Table 3 ) assuming that the $d$-spacing of layers has a more effective role in modulus of elasticity. It means that Sample (3) has not only more $d$-spacing of layers, but also it has more number of layers in stacks than Sample (4) (elastic modulus of Sample (3) is more than of Sample (4)). The variations of yield stress and impact strength also depend on these structural parameters. It seems that the $d$ spacing of layers is more important factor on these properties (compare the result of Figure 3 and Tables 3 and 4). However, in order to understand the relationship between mechanical properties and morphology of samples, it is necessary to investigate the deformation mechanism in different samples. The following section has been allocated to this subject. 

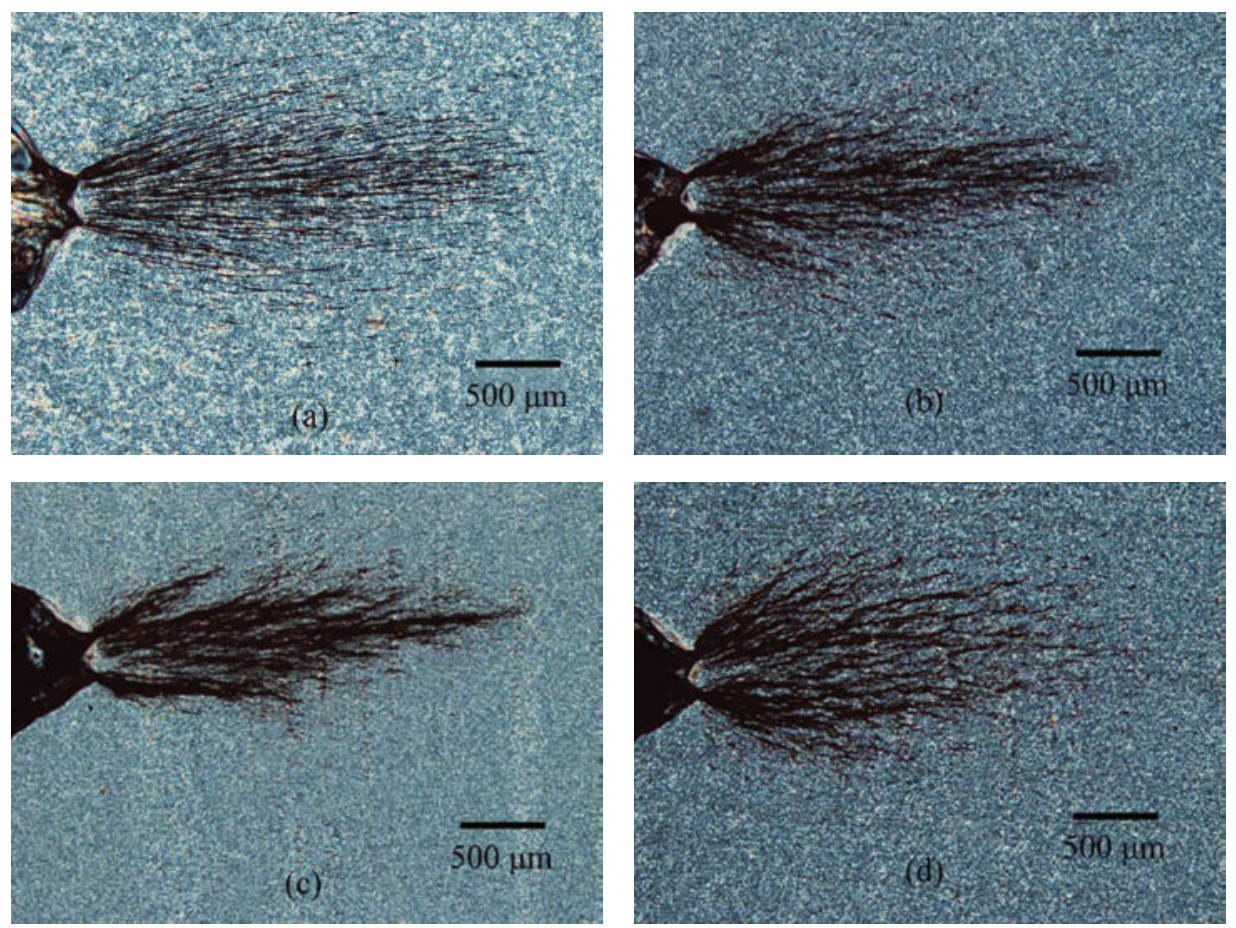

Figure 5: Transmission optical micrographs taken under cross-polarized light showing damage zones at the notch tip of PP/clay without compatibilizer, that is, Sample (2), subjected to $F=215 \mathrm{~N}$ : (a) neat, (b) 1.5 wt.\% clay, (c) 3 wt.\% clay, and (d) 5 wt.\% clay.

3.3. Deformation Mechanism. The mechanisms of deformation at the notch tip of $\mathrm{PP} /$ clay composites without compatibilizer subjected to $3 \mathrm{~PB}$ loading are illustrated in Figure 5.

These micrographs reveal crazing at the notch tips. Similar observation has been reported by Narisawa and Ishikawa [31] and Zebarjad et al. [32]. Figure 5(a) shows individual crazes formed and grown at the notch tip of polypropylene subjected to 3PB loading condition. Addition of organoclay (Figures 5(b)-5(d)) has intensified crazing at the notch tip so that more massive crazing has been observed in those figures. Intensification of crazing at the notch tip in the absence of compatibilizer was observed in our previous work by focusing on a Co-PP [33]. Despite the similar observations made for two studies, that is, intensification of crazing at the notch tip by incorporation of organoclay, there is a difference between the two studies. While the tensile yield stress was improved by adding organoclay in this study (Table 4), the previous work revealed reduction of yield stress by incorporation of silicate layers [33]. It seems that organoclays without compatibilizer tend to delaminate from the matrix while the tension stress is applied. This phenomenon makes the directions of stresses change from shear to normal by incorporating organoclays. As a result, the yield stress of homo-PP increases. In co-PP, some dilatational shear bands initiate and grow around the rubbery phase in the matrix. It is possible that these dilatational shear bands spread in the matrix when they encounter with organoclays which do not have good adhesion to matrix. As a result of this deformation distribution, the yield stress is reduced in co-PP.

In order to investigate the effect of compatibilizer on deformation mechanism, the notch tip of samples was examined (Figure 6).

As observed in Figure 6, incorporation of compatibilizer has reduced the extent of crazing very significantly. Comparing of XRD results (Tables 2 and 3 ) reveals that the $d$-spacing of layers increases by introducing the compatibilizer.

Figure 7 shows the notch tip deformation zones of PP/ clay/PP-g-MA nanocomposites compounded with different feeder ratings from those of Figure 6.

As it was described earlier, the sample compounded at the feeder speed of $15 \mathrm{rpm}$ (Figure 7(b)) has larger $d$-spacing and more numbers of silicate layers per stack in comparison with the other sample (Figure 7(a)). It means that this sample has limited amount of stacks per volume. Because the crazing mechanism tends to initiate and grow by incorporating organoclays (in the form of stacks), the deformation zone (crazing) at its notch tip will be smaller than the other sample under the condition of same loading (the suitable sites for initiation of crazing are less than the other sample).

\section{Conclusion}

Polypropylene/clay nanocomposites via melt intercalation in an intermeshing corotating twin-extruder using starve feeding system were prepared, and these results were obtained. 

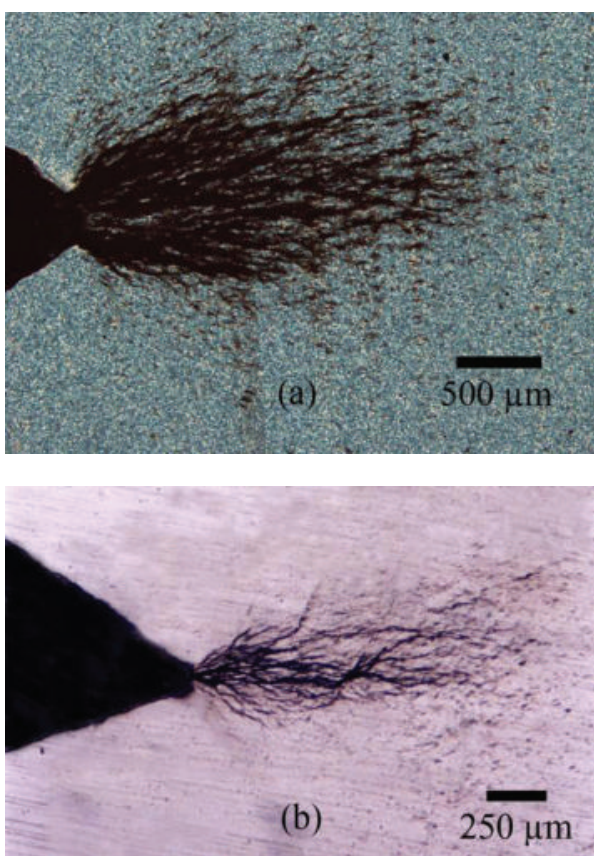

FIGURE 6: Transmission optical micrographs showing damage zones at the notch tip of (a) Sample (1) and (b) Sample (3) subjected to $F=215 \mathrm{~N}$ (samples include $5 \mathrm{wt} . \%$ organoclay and/or $5 \mathrm{wt} . \%$ PPg-MA).

(i) The space between layers has been increased in existence of compatibilizer in both conditions (Rotor Speed $=50$ and $100 \mathrm{rpm}$ ). Of course, due to more residence time, increasing the $d$-spacing of clay in rotor speed of $50 \mathrm{rpm}$ is more than $100 \mathrm{rpm}$.

(ii) The shear rate is more important factor to extend exfoliation than the residence time in the conditions of this experiment.

(iii) With increasing the feeder speed of the extruder the intergallery space and the degree of exfoliation have increased and decreased, respectively.

(iv) The Young's modulus increases with decreasing the rotor speed and the existence of PP-g-MA as compatibilizer. It seems that the $d$-spacing of layers plays more effective role than the amount of layers in each stack, in modulus of elasticity.

(v) With adding the organoclay, the crazing mechanism has changed from local to massive in the mode of $3 \mathrm{~PB}$ loading.

(vi) The deformation zone at the notch tip of samples decreases with increasing the feeder speed.

\section{Acknowledgment}

The authors thank Petrochemical Research and Technology Company (NPC-RT) for funding this research.
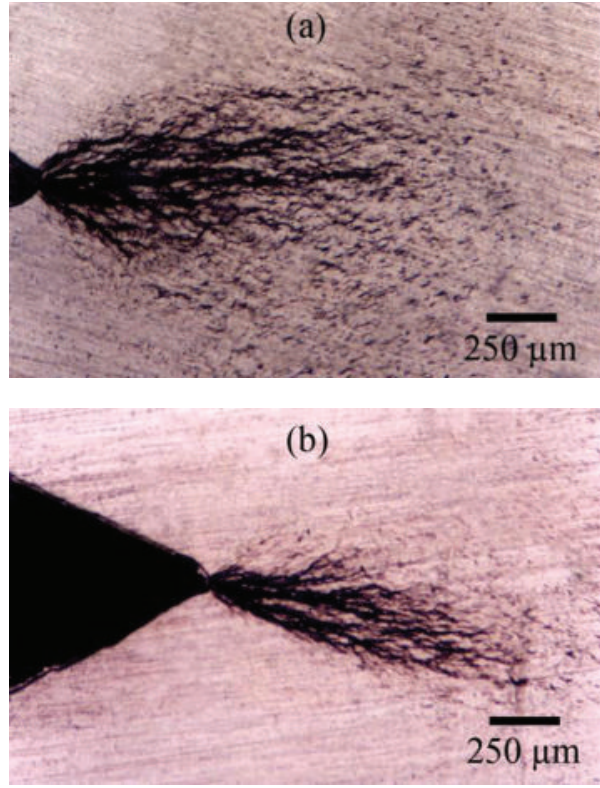

Figure 7: Transmission optical micrographs showing damage zones at the notch tip of PP/5 wt. $\%$ clay $/ 5$ wt. $\%$ PP-g-MA subjected to $F=215 \mathrm{~N}$ : (a) Sample (4), (b) Sample (5).

\section{References}

[1] C.-M. Chan, J. Wu, J.-X. Li, and Y.-K. Cheung, "Polypropylene/calcium carbonate nanocomposites," Polymer, vol. 43, no. 10, pp. 2981-2992, 2002.

[2] E. T. Thostenson, C. Li, and T.-W. Chou, "Nanocomposites in context," Composites Science and Technology, vol. 65, no. 3-4, pp. 491-516, 2005.

[3] J. Jordan, K. I. Jacob, R. Tannenbaum, M. A. Sharaf, and I. Jasiuk, "Experimental trends in polymer nanocomposites- a review," Materials Science and Engineering A, vol. 393, no. 1-2, pp. 1-11, 2005.

[4] Y. Rao and J. M. Pochan, "Mechanics of polymer-clay nanocomposites," Macromolecules, vol. 40, no. 2, pp. 290-296, 2007.

[5] S. S. Ray and M. Okamoto, "Polymer/layered silicate nanocomposites: a review from preparation to processing," Progress in Polymer Science, vol. 28, no. 11, pp. 1539-1641, 2003.

[6] S.-M. Lai, W.-C. Chen, and X.-S. Zhu, "Melt mixed compatibilized polypropylene/clay nanocomposites: part 1-the effect of compatibilizers on optical transmittance and mechanical properties," Composites Part A, vol. 40, no. 6-7, pp. 754-765, 2009.

[7] A. Okada and A. Usuki, "Twenty years of polymer-clay nanocomposites," Macromolecular Materials and Engineering, vol. 291, no. 12, pp. 1449-1567, 2006.

[8] L. Szazdi, A. Pozsgay, and B. Pukanszky, "Factors and processes influencing the reinforcing effect of layered silicates in polymer nanocomposites," European Polymer Journal, vol. 43, no. 2, pp. 345-359, 2007.

[9] R. Abu-Zurayk, E. Harkin-Jones, T. McNally, G. Menary, P. Martin, and C. Armstrong, "Biaxial deformation behavior and mechanical properties of a polypropylene/clay nanocomposite," Composites Science and Technology, vol. 69, no. 10, pp. 1644-1652, 2009. 
[10] H. Palza, R. Vergara, M. Yazdani-Pedram, and R. Quijada, "Polypropylene/clay nanocomposites: effect of different clays and compatibilizers on their morphology," Journal of Applied Polymer Science, vol. 112, no. 3, pp. 1278-1286, 2009.

[11] L. Xu, H. Nakajima, E. Manias, and R. Krishnamoorti, "Tailored nanocomposites of polypropylene with layered silicates," Macromolecules, vol. 42, no. 11, pp. 3795-3803, 2009.

[12] E. Manias, A. Touny, L. Wu, K. Strawhecker, B. Lu, and T. C. Chung, "Polypropylene/montmorillonite nanocomposites. Review of the synthetic routes and materials properties," Chemistry of Materials, vol. 13, no. 10, pp. 3516-3523, 2001.

[13] Y. Kurokawa, H. Yasuda, M. Kashiwagi, and A. Oyo, "Structure and properties of a montmorillonite/polypropylene nanocomposite," Journal of Materials Science Letters, vol. 16, no. 20, pp. 1670-1672, 1997.

[14] M. Kawasumi, N. Hasegawa, M. Kato, A. Usuki, and A. Okada, "Preparation and mechanical properties of polypropylene-clay hybrids," Macromolecules, vol. 30, no. 20, pp. 6333-6338, 1997.

[15] M. Kato, A. Usuki, and A. Okada, "Synthesis of polypropylene oligomer-clay intercalation compounds," Journal of Applied Polymer Science, vol. 66, no. 9, pp. 1781-1785, 1997.

[16] N. Hasegawa, M. Kawasumi, M. Kato, A. Usuki, and A. Okada, "Preparation and mechanical properties of polypropyleneclay hybrids using a maleic anhydride-modified polypropylene oligomer," Journal of Applied Polymer Science, vol. 67, no. 1, pp. 87-92, 1998.

[17] A. Oya, Y. Kurokawa, and H. Yasuda, "Factors controlling mechanical properties of clay mineral/polypropylene nanocomposites," Journal of Materials Science, vol. 35, no. 5, pp. 1045-1050, 2000.

[18] P. Reichert, H. Nitz, S. Klinke, R. Brandsch, R. Thomann, and R. Mülhaupt, "Poly(propylene)/organoclay nanocomposite formation: influence of compatibilizer functionality and organoclay modification," Macromolecular Materials and Engineering, vol. 275, no. 1, pp. 8-17, 2000.

[19] H. Ishida, S. Campbell, and J. Blackwell, "General approach to nanocomposite preparation," Chemistry of Materials, vol. 12, no. 5, pp. 1260-1267, 2000.

[20] Y. Wang, F.-B. Chen, Y.-C. Li, and K.-C. Wu, "Melt processing of polypropylene/clay nanocomposites modified with maleated polypropylene compatibilizers," Composites Part B, vol. 35, no. 2, pp. 111-124, 2004.

[21] H. Wang, C. Zeng, P. Svoboda, and P. James Lee, "Preparation and properties of polypropylene nanocomposites," in Proceedings of the 59th ANTEC Proceedings, pp. 2203-2207, Dallas, Tex, USA, 2001.

[22] K.-N. Kim, H.-S Kimm, and J.-W Lee, "Effect of interlayer structure, matrix viscosity and composition of a functionaiized polymer on the phase structure of polypropylenemontmorillonite nanocomposites," Polymer Engineering \& Science, vol. 41, no. 11, pp. 1963-1969, 2001.

[23] H. R. Dennis, D. L. Hunter, D. Chang et al., "Effect of melt processing conditions on the extent of exfoliation in organoclay-based nanocomposites," Polymer, vol. 42, no. 23, pp. 9513-9522, 2001.

[24] L. Incarnato, P. Scarfato, G. M. Russo, L. di Maio, P. Iannelli, and D. Acierno, "Preparation and characterization of new melt compounded copolyamide nanocomposites," Polymer, vol. 44, no. 16, pp. 4625-4634, 2003.

[25] M. Modesti, A. Lorenzetti, D. Bon, and S. Besco, "Effect of processing conditions on morphology and mechanical properties of compatibilized polypropylene nanocomposites," Polymer, vol. 46, no. 23, pp. 10237-10245, 2005.
[26] W. Lertwimolnun and B. Vergnes, "Influence of compatibilizer and processing conditions on the dispersion of nanoclay in a polypropylene matrix," Polymer, vol. 46, no. 10, pp. 34623471, 2005.

[27] R. J. Bagheri, "Rubber toughened epoxy: roles of particle cavitation and rubber/particle interface," Ph.D. thesis, Lehigh University, Bethlehem, PA, USA, 1995.

[28] C. Rauwendaal, SPC: Statistical Process Control in Injection Molding and Extrusion, Carl Hanser, Munich, Germany, 2000.

[29] Z. Tadmor and C. G. Gogos, Principles of Polymer Processing, John Wiley \& Sons, 1979.

[30] D. G. Baird and D. I. Collias, Polymer Processing: Principles and Design, John Wiley \& Sons, 1998.

[31] I. Narisawa and M. Ishikawa, "Crazing in semicrystalline thermoplastics," in Crazing in Polymers (Advances in Polymer Science, Vols 91/92), H. Kausch, Ed., p. 353, Springer, Berlin, Germany, 1990.

[32] S. M. Zebarjad, R. J. Bagheri, A. Lazzeri, and S. Serajzadeh, "Fracture behaviour of isotactic polypropylene under static loading condition," Materials and Design, vol. 24, no. 2, pp. 105-109, 2003.

[33] B. Akbari and R. J. Bagheri, "Influence of PP-g-MA on morphology, mechanical properties and deformation mechanism of copolypropylene/clay nanocomposite," Journal of Applied Polymer Science, vol. 114, no. 6, pp. 3751-3759, 2009. 

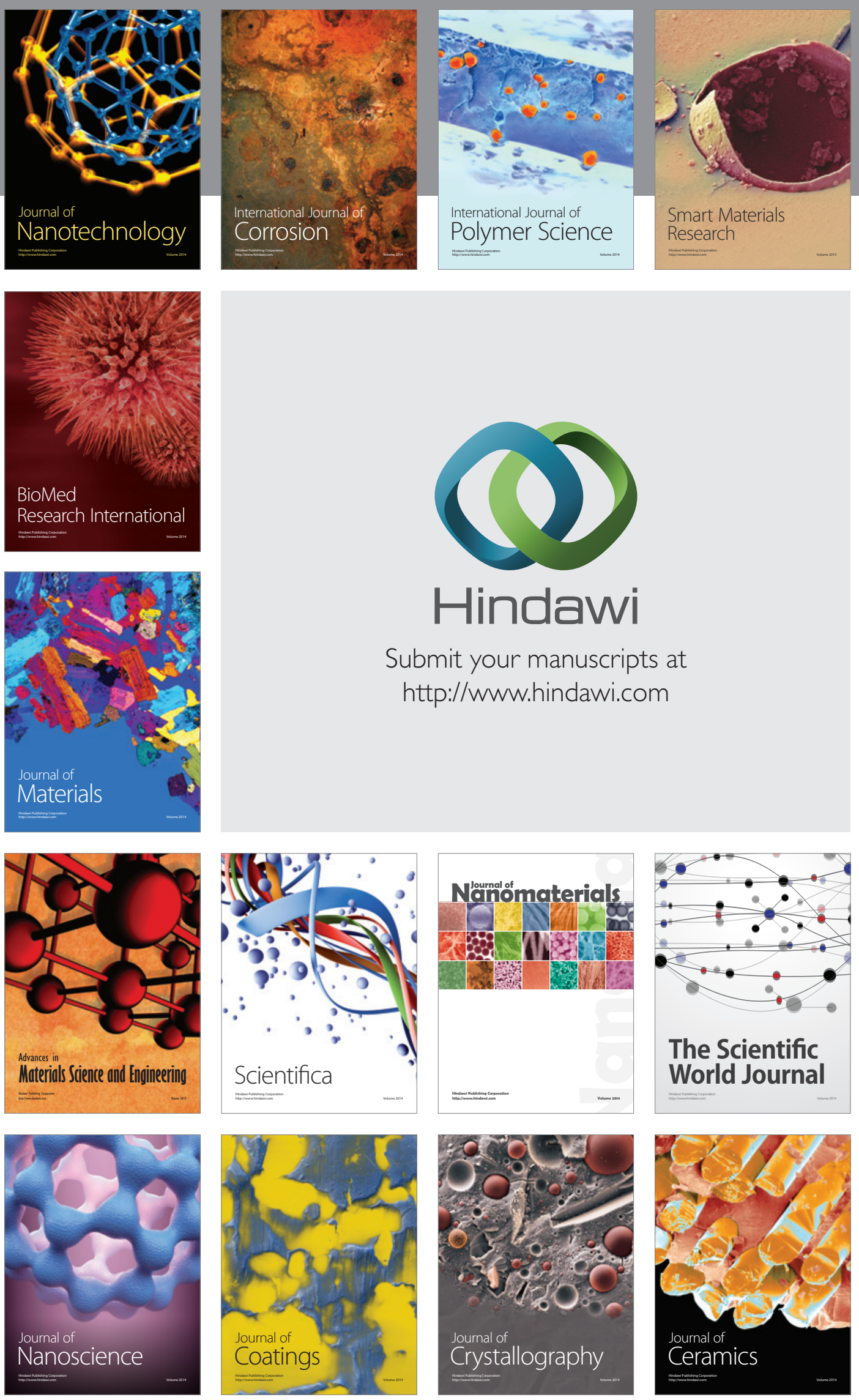

The Scientific World Journal

Submit your manuscripts at

http://www.hindawi.com

\section{World Journal}

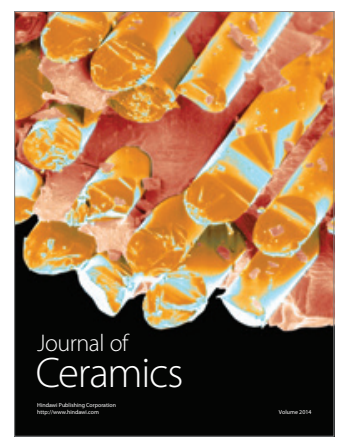

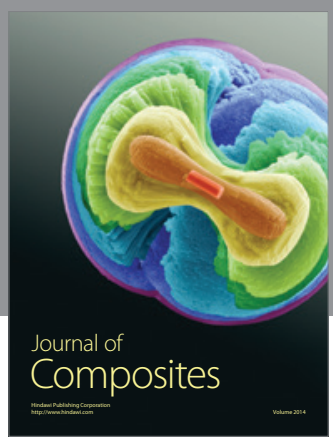
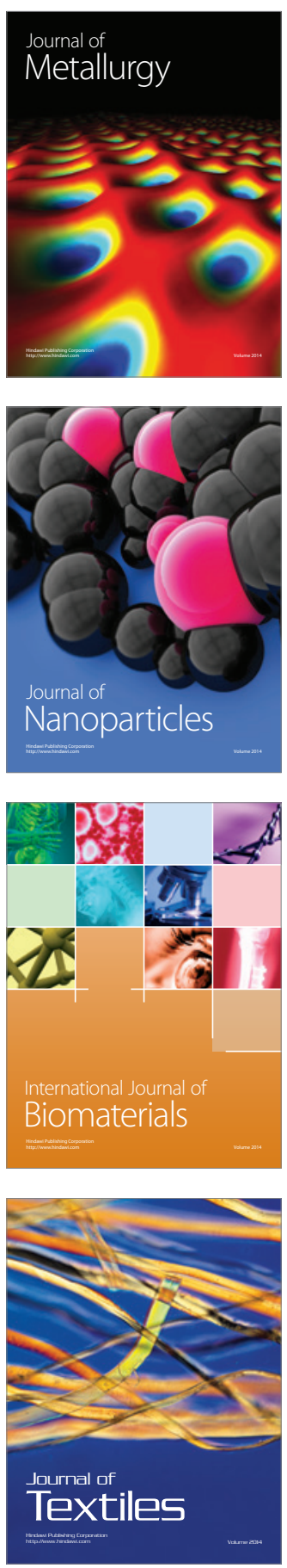